\title{
Why They Still Cannot Use Their Library Catalogues
}

\author{
Peter Warren \\ University of Natal, South Africa
}

\author{
peterw@cs.unp.ac.za
}

\begin{abstract}
The paper examines the Urica World Wide Web Online Public Access Catalogue (OPAC) from three different perspectives. The heuristic evaluation technique showed up a number of problems at the detail level. For broader concerns this needed to be supplemented by other approaches. The use of user interface design patterns and Laurel's concepts of Computers as Theatre showed up the fact that the design needs to be more object oriented with the tools secondary and the catalogue primary. This problem applies to many library catalogues and not just Urica. One limitation of the current design patterns is they provide little help with aesthetic issues. No one technique for evaluating user interfaces is sufficient as problems range from the micro to the macro level.
\end{abstract}

Keywords. Online public access catalogues, OPAC, heuristic evaluation, patterns, human computer interaction.

\section{Introduction}

A university library represents a considerable, critical, and very expensive information resource. It is convenient, archived over a long period of time and available to all members of the institution. By way of contrast electronic media tend to be temporal and available only to subscribers. Print media also receive the overwhelming number of citations. But if the information is not accessible, this costly investment is not going to be realized to the full. In addition the library has to compete with other information resources, possibly inferior, but if perceived to be more convenient they will be utilized in preference. Furthermore, poor usability of the human computer interface of any system can drive up training and support costs; a situation that cash strapped educational institutions can ill afford. Failure, or less than optimal usability, of library catalogues, therefore, can have serious implications for tertiary educational institutions as a whole. A positive development is the emergence of OPACs (Online Public Access Catalogues) via World Wide Web (WWW) browsers such as Netscape and Internet Explorer because this potentially provides wider access. But how good are these catalogues? It is not enough for them to be as good as previous meth-

Material published as part of this journal, either on-line or in print, is copyrighted by the publisher of Informing Science. Permission to make digital or paper copy of part or all of these works for personal or classroom use is granted without fee provided that the copies are not made or distributed for profit or commercial advantage AND that copies 1) bear this notice in full and 2) give the full citation on the first page. It is permissible to abstract these works so long as credit is given. To copy in all other cases or to republish or to post on a server or to redistribute to lists requires specific permission and payment of a fee. Contact Editor@inform.nu to reauest redistribution bermission. ods, they will have to be easier to use in order to compete with WWW products and other electronic methods. Conversely it maybe premature to consider virtual libraries if we cannot even get our conventional library catalogues to be adequately usable. The literature on computer based OPACs is not encouraging showing that few users are able to recover the information they need, and that the heaviest class of users are the most at risk of not being able to use their catalogues. Usability aspects of WWW based OPACs are thus of substantial importance to the emerging spectrum of possibilities that scholars will have available to them in their search for information.

This paper concentrates on one particular OPAC namely the Urica system, which is widely used in South African higher education. An analysis is made of the user interface of this system from three points of view. Firstly a method called heuristic evaluation (e.g. Nielsen \& Mack, 1994) is employed which compares the interface with published user interface guidelines. A more recent development has been the use of "patterns" as a method of designing user interfaces (Tidwell, 1999). Patterns are "solutions in context" where a particular emphasis is placed upon the resolving of the various forces that influence a design. Finally we consider a more unconventional approach to user interface design via Laurel's (1993) theories of Computers as Theatre. This will be supported by actual observations of library patron behavior reported in the literature. We will demonstrate that all three of these techniques show that the Urica OPAC has several deficiencies that could relatively easily be corrected. Some of these problems are also found in other OPACs. Techniques such as reducing the number of screens, taking a more user centered ap- 
proach to vocabulary employed, better help facilities and even simple aesthetic changes are modifications that can have a substantial influence on the usability of an OPAC and are the kind of recommendations that this research suggests. Before we conclude that more esoteric approaches are needed to the catalogue these elementary changes in user interface design need to be implemented.

\section{Heuristic Evaluation}

Heuristic evaluation is a term introduced by Nielsen \& Mack (1994). It is a process by which developers, as opposed to human computer interaction (HCI) experts, evaluate an interface against a series of guidelines or heuristics, and hence the name. The claimed advantage is that it is cheap to implement, and that it is better to perform a heuristic evaluation than to do nothing at all. An heuristic evaluation is one of a number of methods called "usability inspection techniques". The alternative is to observe patrons using the system and such methods are called "empirical evaluations". It might be felt that the empirical study is the "acid test" but this is not so, as such methods can also fail to find faults. The point is made explicitly by Wharton et al. (1991), and we quote :- "there were many serious problems that it (empirical evaluation) failed to find". We decided to use a hybrid of both approaches. A study of the Urica WWW OPAC was carried out by two honours students (de Witt \& Norton, 1999) with this author acting as advisor. To the extent that guidelines were used (but not the original guidelines) and that the two students were not HCI experts, the term heuristic evaluation is appropriate to describe the protocol used even if some empirical techniques were employed. The use of guidelines strengthens the case that the problems found were real and not just taste issues. The evaluation was supplemented by using a questionnaire while at the same time observing patrons using the OPAC. In a sense the questionnaire resulted in the patrons becoming additional evaluators. The two investigators are particularly frank about the problems they had with their methodology in particular the refusal of some patrons to assist. These problems, and the fact that a non-standard protocol was used, will make it difficult to compare with other results using heuristic evaluation, however it will not invalidate the reality of problems that were uncovered. The evaluation took place on a detailed screen-by-screen inspection of the OPAC. It is not the place to reproduce all these detailed results but the overall conclusions are well justified. The evaluators found that Urica had a number of good points but that the general design hindered rather than helped the patrons. In fact the interface performed badly in half the usability heuristics presented by Nielsen \& Mack (1994). Particularly important factors were:-

- Navigation was made difficult by the inability to move easily between screens, the lack of exit points and having too many screens.

- The interface was not aesthetically pleasing. This is important as aesthetics improves readability and thus usability.

- Library jargon decreased understanding. Interestingly one of the examiners (Kaniki, 1999) in the field of Library Science objected to this conclusion and felt that patrons should be educated in library terminology. Nevertheless "speak the users' language" is a key heuristic in Nielsen \& Mack (1994).

- The help system, when available, was difficult to scan and extract information from.

- The interface had many problems at a detailed level resulting from layout and gratuitous items. Especially irritating was a flashing line serving no particular purpose.

There was also an evaluation of three other WWW OPAC systems (University of the Witwatersrand, Oxford University and The Library of Congress). They found that the aesthetics of these systems is superior, there is a greater density of information on the screens and the support for navigation is better. All of these issues lead to greater usability of the competing systems. However these OPACs worked in much the same way as Urica in that they went through a series of menus to reach the catalogue. The question is whether this is the right approach. The evaluations would not be able answer this question as they were done on a screen-by-screen basis. We experienced a limitation of the heuristic evaluation technique in that it tended to concentrate on micro features rather than the global picture. Other methods of evaluation are needed to answer the question of whether a series of menus is the correct approach.

\section{A Critique Using Patterns}

Patterns is a term invented by the architect Christopher Alexander (1979) who was looking for a generative approach to creating beautiful buildings and cities. He looked at the most effective structures from the past and attempted to find out what made them so. His conclusions (if it is possible to paraphrase Alexander) were that the most ef- 


\section{Why they still cannot use their library catalogues}

fective designers used a series of patterns to generate the design. The idea of patterns is that they provide solutions in context as opposed to guidelines, which tend to state 'you do it this way' in all circumstances. A number of research workers have experimented with patterns in software and there is an increasing interest in the field of human computer interaction (HCI) as well. They seem particularly appropriate in this area as HCI combines both the technical and aesthetic issues in much the same way that architecture does. There have been a number of attempts to produce patterns for HCI, many of them only reported on the Web. The pattern set developed by Jenifer Tidwell (1999) has been widely cited despite never having been subjected to a peer review process. If patterns are useful, and Tidwell's patterns, in particular, are useful, they should be able to be used to evaluate in well as generate user interfaces. The objective here is thus to both evaluate the usefulness of Tidwell's patterns and whether they are consistent with the Urica interface. Tidwell appears much influenced by Laurel's (1993) thought provoking book and sees effective artifacts as having the following properties (and we quote): -

1. They shape the user's understanding of something, through a stylized presentation that unfolds the content to the user in an appropriate way. A successful artifact will enable its users to completely understand and effectively use the content being presented.

2. They enable a user to accomplish a task, by progressively unfolding the action possibilities to the user at an appropriate pace as the user interacts with it. A successful artifact will "flow" so well that it lets its users focus entirely upon the task at hand, causing the artifact itself to fade from the user's awareness.

While no one is likely to dispute these lofty ideals, it might be considered difficult to apply them in practice. It requires a more holistic approach than software developers are normally accustomed. When we try, however, we get some useful results. Is the step-by-step process that Urica uses to guide the patron to the answer "progressively unfolding the action possibilities to the user at an appropriate pace as the user interacts with it"? We think not. We think that the approach is a rigid straight jacket that forces the patron into a certain way of searching and where the patron focuses on the tool and not the catalogue. This is quite the opposite of "the artifact fading from the user's awareness". To meet the requirements the patron would have to see the catalogue at all times and then be able to manipulate it to suite the patrons needs. Such an approach is known as an object oriented user interface (Collins, 1995). As we move to the details, Tidwell sees her pattern language as consisting of a number of sub-languages. By looking at the contexts and forces in these, we should be able to decide if the Urica designers have made the appropriate choices. The patterns that are relevant are: -

- Step-by-step Instructions.

- Composed command

- High-density information display.

- Form.

- Navigable spaces.

Step-by-step instructions. The Urica system takes the user through a series of web pages, which are essentially menus. First the patron is asked to decide which part of the catalogue s/he wishes to search, then the kind of search and only then is an input field used. In this sense Urica uses the "step-by-step instructions" pattern. The pattern suggests that this approach is used when there is limited time, knowledge or (screen) space. The method is known to irritate experienced users. The forces that would lead to the adoption of the step-by-step pattern are trying to avoid burdening the user with options s/he would never use, fear, on the part of the use about doing the wrong thing, and an inability to automate the steps because user input is required. This author contends that these forces are not present and that the choice of this approach is inconsistent with the pattern usage. The Urica implementation lacks an effective navigation scheme, which is also required when using the pattern. This supports the difficulties with navigation found in the heuristic evaluation.

Composed command. Composed commands are similar to the type of interaction used by UNIX or MSDOS. The pattern suggests that they be used for experts, when actions cannot be used graphically, when there is no visual channel (eg in telephone interfaces) or when feedback on the correctness of commands can be given. Three of these four issues indicate that the designers of the OPAC were wise to eschew the composed command.

High-density information display. While more appropriate to the visualization of numeric data, this pattern is also strongly suggested when the user has to find small amounts of information in a mass of other data. This is typical of a library catalogue. Hierarchies and the importance of navi- 
gation between screens are emphasized in the pattern because there is too much information to present at once. The Urica OPAC does not do this and the information content of each screen is somewhat poor. The pattern is indicated but the Urica catalogue makes no use of it.

Form. Urica does use forms to solicit information from the user but it could place much of the step-by-step instruction approach onto a single form. Thus, although forms are used, Urica does not use them correctly.

Navigable spaces. There is far too much information to place on a single page, and thus the user needs to navigate from one page to the next. The pattern makes it clear that this is extremely important. Urica is very weak in this respect and informal demonstrations easily show how the patron can get lost in cyber space. The pattern "Go back to a safe place" would have helped enormously.

Where patterns are not helping. A particularly delightful book on the subject of "Designing Visual Interfaces" by Mullet \& Sano (1995) gives much advise on how to use visual information effectively. Very little of that kind of material can be found in Tidwell and as a result it is not possible to analyze the aesthetic issues in an interface using her patterns. An extension of them in this direction seems desirable.

\section{Conclusions from patterns}

Advice on the way the action should unfold, on navigable spaces, on the inappropriateness of step-by-step instructions, if applied would have greatly improved the Urica system. There are also a number of more detailed techniques in her patterns, not discussed here, that might have further improved the interface. Nevertheless the use of patterns still required some level of judgment and did not incorporate assistance on aesthetic issues. Patterns are useful but not a panacea.

\section{Laurel's Views}

Brenda Laurel (1993) has produced a thought-provoking book "Computers as Theatre" where she considers Aristotle's views on theatre and applies them to the construction of computer programs. While this may appear somewhat fanciful she is at pains to point out that both disciplines are involved in the representation of real world objects in an artificial environment. Like Alexander, Laurel is couched in artistic terms that make the ideas of both them hard to apply especially for technologists. However some of her concepts about dramatic action can be interpreted in our library context with some interesting consequences.

The Flying Wedge. The term flying wedge is a term that Laurel uses to describe the progress of a play. At the start of the drama everything is possible while at the end all possibilities are exhausted except the one. We can apply this concept to the search for information. At the start of the process the whole library catalogue is at our disposal while at the end we have cut the process down to the few documents that will be useful to the patron. But this is not the way most library catalogues work. A patron normally issues a series of commands to the system, and when that fails, the patron issues a new series of commands unrelated to the first. The system provides no help in the continuity of the process. Laurel talks of this as the computer being seen as a tool, so the tool becomes uppermost and the result secondary. Borgman (1996), coming from a completely different direction, mirrors this concern as many patrons search for information over several sessions. To see these sessions as disjoint rather than connected fails to mirror the patrons' view of the wholeness of the interaction.

Causality. Causality refers to the cause and effect relationships within the action that is being represented. Of particular concern to Laurel is the harm that gratuitous incidents have on the ability to understand the action. The main problem with Urica in this context is the poor artwork on the screen. The advertisement for the package is more prominent than the instruction to the user. The borders around the boxes are too heavy, distracting the patron from the main purpose of the activity. The flashing line is immediately commented upon as distracting by students asked to evaluate the interface. Particularly unfortunate in this context are the poor navigation aids available to the patron. A most confusing case is the 'Back' button on the title search form, which takes one to the advanced search screen and not the previous search screen. Causality mirrors concerns found in the patterns and heuristic evaluation approaches.

Shape. Laurel quotes Freytag's analysis where plays rise to a climax and then dies away again. Laurel fortunately helps us in understanding how to apply this by referring to a spreadsheet example. By analogy with this one might expect that an exploration process will take place with the library catalogue. This process will move through the steps of "discover, surprise and reversal" as the patron encounters unexpected information resources, and is disappointed 


\section{Why they still cannot use their library catalogues}

by some expected sources are not available or appropriate. Urica does not support this.

\section{Conclusions from Laurel's work}

Whether drama is serious enough to apply to computer programs is discussed by Laurel herself. Our contention here is that the concepts are applicable to the analysis of Urica and that Urica is not consistent with these concepts. Whether Urica is the worse for it is more difficult to establish. Borgman's (1996) arrival at the same answer from another direction for at least one of the concepts, and Laurel's own analysis, is cause for believing that the analysis is correct and that Urica is deficient.

\section{Conclusions}

Why can they still not use their library catalogues? The situation is clear enough. The Urica catalogue, and others, show a number of errors in human computer interaction techniques. The problems show up at a micro level as well as at a more fundamental level in how the macro interaction takes place. The analysis shows that these errors are relatively easily found and that the problems are caused by rather elementary blunders stemming from not applying human interaction theory correctly. It is contended that many of these errors are relatively easily fixed. The analysis by de Witt \& Norton makes it clear that the lower level problems of screen layout and navigation are not as evident in the three other OPACs evaluated. The upper level concerns about how the interaction proceeds, however, are much the same in these systems. We contend, therefore, that much can be done at a relatively low cost to improve the usability of especially the Urica WWW catalogue system. We do not think that university administrations should put up with poor interfaces. It will cost them dearly. We look forward to online catalogues becoming much easier to use and we do not think that too much effort need be expended in getting there.

The work is also of significance in that it combines three techniques and draws upon ideas going back as far as Aristotle combining both scientific and artistic approaches. The conclusions both overlap and complement each other as well as being consistent with conclusions drawn by library science. We trust it is not too dramatic a conclusion that to "inform science" a wide range of techniques is useful. At least that is what we found even in the mundane matter of library catalogues.

\section{References}

Alexander, Christopher. (1979). The Timeless Way of Building. New York: Oxford University Press.

Borgman, Christine L. (1996). Why are online catalogs still hard to use? Journal of the American Society for Information Science, 47, 493503.

Collins, Dave. (1995). Designing object-oriented user interfaces. Redwood City, CA: Benjamin/Cummings.

de Witt, D., \& Norton, J. (1999). UNP WWW OPAC Prototype. Technical report, University of Natal, Pietermaritzburg.

Kaniki, Andrew. (1999). Examiners report on honour's project by de Witt \& Norton (1999). Private Communication.

Laurel, Brenda. (1993). Computers as Theatre. Reading MA: AddisonWesley.

Mullet, Kevin, \& Sano, Darrell. (1995). Designing Visual Interfaces. Englewood Cliffs, NJ: Sun Soft Press (Prentice Hall).

Nielsen, J., \& Mack, R. Eds. (1994). Usability Inspection Methods. New York: Wiley.

Wharton, C., Jeffries, R., J. Miller, J., \& Uyeda, K. (1991). User interface evaluation in the real world: A comparison of four techniques. Proceedings ACM CHI'91 Conference (New Orleans, L.A., April 28 - May 2), 119-124,

Tidwell, Jenifer. (1999). Common Ground: A pattern language for human computer interface design. [ttp://Ww. mit.edu/ jtidwell/common_ground.html. (ס Jan 2001)

\section{Biography}

Peter Warren is a professor in the Information Technology Programme at the University of Natal in South Africa. He holds a BSc(Honours) in Physics from Natal and a PhD in Astrophysics from Cambridge. He has been involved in IT since 1980 at the University of Port Elizabeth and later back to his alma mater at the University of Natal. He has a special interest in human computer interaction and programming. 